\title{
Norepinephrine was superior in death risk reducing and hemodynamics compared to dopamine in treatment of patients with septic shock
}

https://doi.org/10.1515/pteridines-2021-0002

received October 25, 2020; accepted December 11, 2020.

\begin{abstract}
:
Background: To investigate the clinical effects of norepinephrine versus dopamine in treatment of septic shock by pooling the data form open published clinical trials.
\end{abstract}

Material and Methods: The clinical trials relevant to norepinephrine versus dopamine in treatment of septic shock were electronically searched in the databases of Pubmed, Embase, the Cochrane Library, Web of Science, Google scholar and CNKI. The original data related to the treatment effects such as death risk, oxygen metabolism and hemodynamics index were extracted from the included original studies. The death risk was pooled by the effect size of relative risk (RR), the oxygen metabolism and hemodynamics index were pooled by standard mean difference (SMD) and the corresponding 95\% confidence interval (95\%CI). The publication bias was evaluated by Begg's funnel plot and Egger's line regression test.

Results: Thirteen clinical trials were included in the meta-analysis. The pooled results demonstrated the death risk was significantly decreased ( $\mathrm{RR}=0.89,95 \% \mathrm{CI}: 0.81$ to $0.98, p=0.024$ ) in septic shock patients who received norepinephrine compared to those receiving dopamine. The HR (SMD $=-1.84,95 \% \mathrm{CI}:-2.86$ to $-0.81, \mathrm{p}<0.01$ ) and cardiac index (SMD $=-0.74,95 \% \mathrm{CI}:-1.01$ to $-0.48, \mathrm{p}<0.01)$ were lower in norepinephrine group compared to dopamine group. The systemic vascular resistance index $(\mathrm{SMD}=1.33,95 \% \mathrm{CI}: 0.62$ to $2.04, \mathrm{p}<0.01)$ in norepinephrine group was higher than those of dopamine group with statistical difference. The Begg's funnel plot and Egger's

\footnotetext{
*Corresponding author: Yueying Wu, Department of Emergency, Lishui People's Hospital, $6^{\text {th }}$ Affiliated Hospital of Wenzhou Medical University, Lishui Zhejiang 323000 PR China, E-mail: 1261634597@qq.com Xudong Lu, Xianghua Xu, Department of Emergency, Lishui People's Hospital, $6^{\text {th }}$ Affiliated Hospital of Wenzhou Medical University, Lishui Zhejiang 323000 PR China
}

line regression test $(\mathrm{t}=-0.84, \mathrm{p}=0.425)$ showed no publication bias.

Conclusions: Based on the present evidence, norepinephrine was superior to dopamine in the aspects of death risk reducing and hemodynamics.

Keywords: Clinical effects; norepinephrine; dopamine; septic shock; meta-analysis.

\section{Introduction}

Sepsis refers to the organ dysfunction, which is lifethreatening due to the maladjustment of host response caused by infection [1]. Septic shock is a form of sepsis which is seriously and life threatening. Early diagnosis and treatment of life threatening septic shock are extremely important for improving the patients' prognosis and survival [2]. At present, the concept of multi-dimensional intensive therapy is most accept and practiced clinically, and vasoactive drugs are important part of intensive therapy [3]. However, the mortality of septic shock was still high despite the applecation of intensive treatment, including vasoactive drugs, volume resuscitation, effective anti-infection, mechanical ventilation etc. Norepinephrine and dopamine have been used as the first-line drugs in the treatment of septic shock, but their clinical efficacy were controversial according to the results of previous publications. Although some studies have pointed out that norepinephrine can improve hemodynamics and reduce mortality of septic shock patients more than dopamine, other studies have also shown that norepinephrine can aggravate organ ischemia, and it can't improve the prognosis of septic shock patients compared with dopamine. In year 2012, a meta-analysis relevant to norepinephrine versus dopamine in treatment of septic shock indicated that the death risk was significant decreased in patients received norepinephrine compared to dopamine [4]. However, this study didn't evaluate the 
hemodynamic and metabolic parameters between the two drugs. In year 2017, Belletti and his colleges [5] performed a network meta-analysis the effect of vasoactive drugs on mortality in patients with severe sepsis and septic shock. They found that norepinephrine improved survival compared with dopamine (OR, 0.81; 95\% CI, 0.66-1.00). However, the paper didn't compare other parameters such as heart rate, blood lactic acid, oxygen consumption and oxygen delivery index. Several years had passed since the previous meta-analysis relevant to norepinephrine versus dopamine in treatment of septic shock published, there were several new clinical trials of norepinephrine versus dopamine in treatment of patients with septic shock published. Therefore, we performed this meat-analysis by adding the new publications to further comparing the clinical efficacy of norepinephrine versus dopamine in treatment of septic shock in order to provided more evidence for septic shock management.

\section{Material and Methods}

\section{Studies electronic screening}

The clinical trials relevant to norepinephrine versus dopamine in treatment of septic shock were electronically searched in the databases of Pubmed, Embase, the Cochrane Library, Web of Science, Google scholar and CNKI. The studies were searched in the electronic databases by two reviewers independently and made cross checking. The electronic searching keywords were: "norepinephrine", "dopamine", "septic shock". The references of the related studies were also screened in order to identify the potential suitable publications.

\section{Studies inclusion and data extraction}

The study inclusion and exclusion criteria was were constructed in the aspects of study design, patients selection of the included original study, intervention methods and outcomes. For inclusion criteria, the studies should fulfill the below conditions: 1)The study was prospective random clinical trials; 2) The cases in each original study should be clearly diagnosed of septic shock with the age more than 18 years old; 3) Patients received either norepinephrine or dopamine for the treatment of septic shock; 4) At least one of the below measurements was provided in the original study(1)Death risk; (2) Heart rat; (3)Cardiac index; (4)MAP; (5)Systemic vascular resistance index;(6)Blood lactic acid; (7)Oxygen consumption; (8) Oxygen delivery); 5)The publication languages were limited to English or Chinese.

The study exclusion criteria was: 1)Case report, literature review or retrospective observation study; 2) Shock callused by other disease such as traumatic or cardiogenic; 3) Other treatment drugs than norepinephrine or dopamine; 4)None of the outcomes related to (1)Death risk; (2)Heart rat; (3)Cardiac index; (4)MAP; (5)Systemic vascular resistance index; (6)Blood lactic acid; (7) Oxygen consumption; (8)Oxygen delivery was provided in the original study; 5) Duplicated published data or study.

\section{Ethical approval}

The conducted research is not related to either human or animals use.

\section{Quality assessment}

The methodical quality of the included 13 clinical trials were evaluated by the Jadad score [6] which containing the questionnaire of: Adequate sequence generation?; Allocation concealment?; blinding? ; Withdraw?. The methodology quality was low if the score $<2$, otherwise was considered high methodical quality for the included studies.

\section{Statistical analysis}

STATA 12.0 software was applied for data analysis. Death risk was pooled by relative risk (RR). The oxygen metabolism and hemodynamics index were pooled by standard mean difference (SMD) and the corresponding 95\% confidence interval (95\%CI). Publication bias was evaluated through Begg's funnel plot and Egger's line regression test. Two tails $\mathrm{p}<0.05$ was deemed as statistical difference.

\section{Results}

\section{Publications searching}

After systematic electronic searching the database, 827 relevant publications were initially identified. 798 studies were further screened by the title and abstract in order 
Table 1: The general characteristics of the included publications.

\begin{tabular}{|c|c|c|c|c|c|c|c|}
\hline Author & Year & No. & Age(Year) & APACHEII & $\mathrm{BP}(\mathrm{mmHg})$ & Jadad score & Out come \\
\hline Wu YF[7] & 2016 & 32 & 48.9 & NA & $M A P<80$ & 2 & (2)(3) (4) \\
\hline He HW[8] & 2012 & 80 & NA & NA & $\begin{array}{l}\mathrm{MAP}<60 \text { or } \\
\mathrm{SBP}<90\end{array}$ & 2 & \\
\hline Zhuang YG[9] & 2011 & 80 & 55.0 & 24.9 & $M A P<60$ & 2 & (1)(2)(3) (4) \\
\hline Agrawal A[10] & 2011 & 50 & $54.6 / 52.8$ & 24.6 & $\mathrm{SBP}<90$ & 3 & (2) (3) (4) (5) 7 (8) \\
\hline Wu JZ[11] & 2010 & 46 & 55.5 & 21.8 & $M A P<60$ & 2 & (1) \\
\hline De Backer[12] & 2010 & 1044 & 67.5 & NA & $M A P<70$ & 5 & (1) \\
\hline Liu P[13] & 2010 & 50 & 43.2 & 17.3 & $M A P<60$ & 2 & (1) 6 \\
\hline Patel[14] & 2010 & 252 & NA & 27.5 & $\begin{array}{l}\mathrm{MAP}<60 \text { or } \\
\mathrm{SBP}<90\end{array}$ & 2 & (1) \\
\hline Gao JL[15] & 2008 & 44 & 56.0 & NA & $\mathrm{SBP}<90$ & 1 & (1) 8 \\
\hline Mathur & 2007 & 50 & 53.7 & 25.1 & $\mathrm{SBP}=75.6$ & 3 & (1)(2)(3) (4) \\
\hline Marik[16] & 1994 & 20 & 46.0 & 17.5 & $M A P<60$ & 3 & (1)(2)(3)(4)(5)(6) (7) \\
\hline Martin[17] & 1993 & 32 & 52.5 & 30.5 & $\mathrm{SBP}<90$ & 2 & (1)(2)(3)(4)(5)(6)(8) \\
\hline Ruokonen[18] & 1993 & 10 & 45.1 & 13.3 & $S B P<90$ & 3 & (1)(2)(3) (4) (5) (6) (8) \\
\hline
\end{tabular}

Notes: (1)Death risk; (2) Heart rat; (3)Cardiac index; (4)MAP; (5)Systemic vascular resistance index; (6)Blood lactic acid; (7) Oxygen consumption; 8) Oxygen delivery

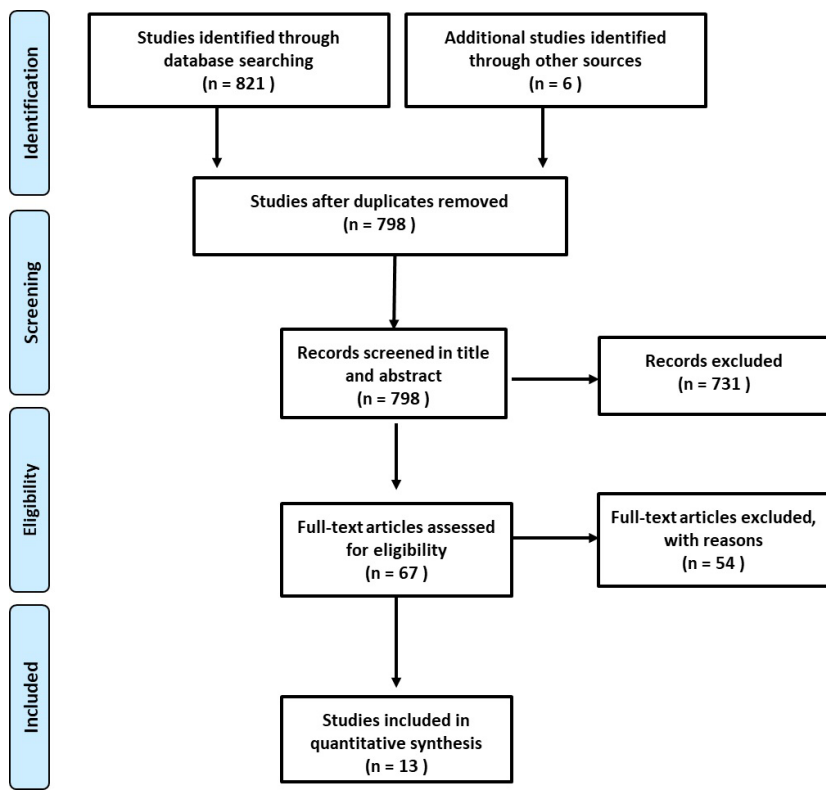

Figure 1: The studies electronic searching flow chart.

to eliminate the duplicated publications or data. Finally, 13 clinical trials[7-18] relevant to norepinephrine versus dopamine in treatment of septic shock were included in the meta-analysis. Figure 1.

\section{General quality of the included studies}

Thirteen clinical trials of norepinephrine versus dopamine in treatment of septic shock with 1790 cases were included in the preset work. The age of the included subjects ranged from 43.2 to 67.5. The APACHEII score ranged from 13.3 to 30.5 with median of 23.2. The MAP of the included studies was more than $60 \mathrm{mmHg}$. The methodical quality score (Jadad) ranged from 1 to 5 with the median of 2.46 . The main characteristics of the included 13 studies were showed in Table 1.

\section{Pooled death risk}

The death risk was pooled through fixed effect model between norepinephrine and dopamine in treatment of septic shock due to no statistical heterogeneity $\left(\mathrm{I}^{2}=0.0 \%\right.$, $\mathrm{p}=0.964$ ). The pooled result demonstrated that the death risk was significant decreased ( $R R=0.89,95 \% \mathrm{CI}: 0.81$ to $0.98, \mathrm{p}=0.024$ ) in septic shock patients who received norepinephrine compared to those receiving dopamine, Figure 2. 


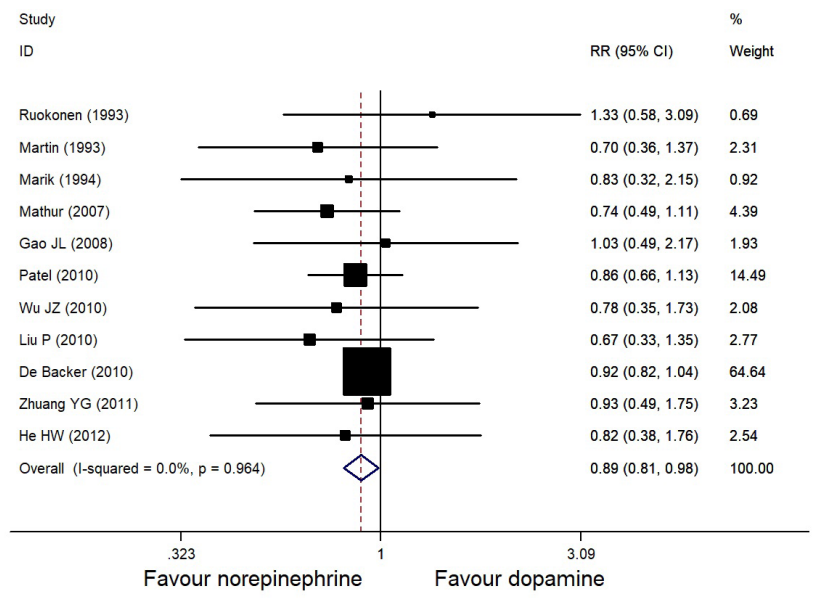

Figure 2: The forest plot of relative risk of death for norepinephrine versus dopamine in treatment of septic shock.

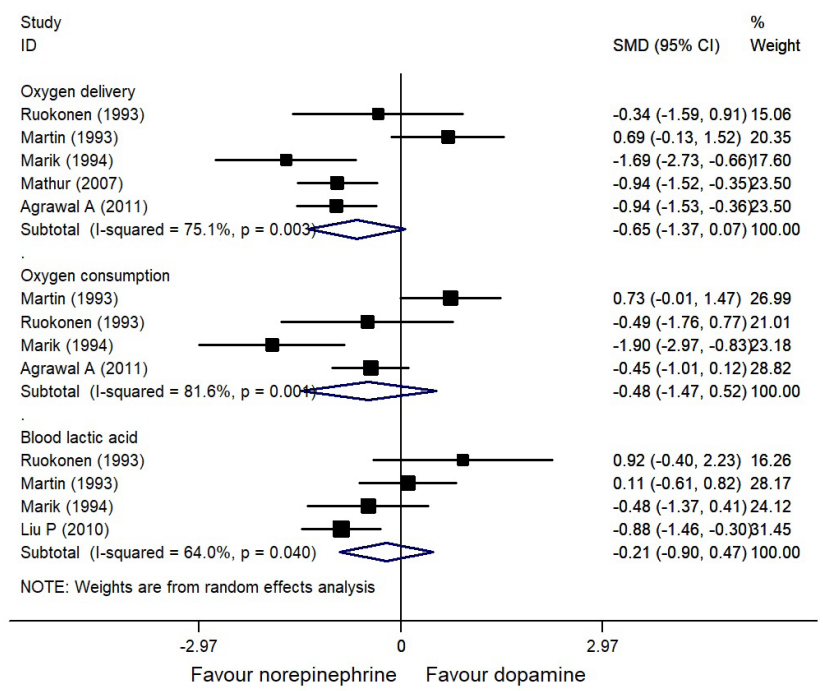

Figure 3: The forest plot of Pooled oxygen metabolism index for norepinephrine versus dopamine in treatment of septic shock.

\section{Pooled oxygen metabolism}

Blood lactic acid, oxygen consumption and oxygen delivery index were pooled under random effect mode because of significant statistical heterogeneity $\left(\mathrm{I}^{2}>50 \%\right.$, $\mathrm{p}<0.05)$. The pooled results indicated that there were no statistical difference for blood lactic acid ( $\mathrm{SMD}=-0.21$, 95\%CI:-0.90 to $0.47, \mathrm{p}=0.54$ ), oxygen consumption (SMD=$0.48,95 \%$ CI:-1.47 to $0.52, \mathrm{p}=0.35$ ) and oxygen delivery index (SMD $=-0.65,95 \%$ CI:-1.37 to 0.07, $\mathrm{p}=0.08$ ) between norepinephrine and dopamine in treatment of septic shock, Figure 3.

\section{Pooled hemodynamics index}

The pooled results indicated that the HR (SMD=1.84, 95\%CI:-2.86 to $-0.81, \mathrm{p}<0.01$ ) and cardiac index (SMD=-0.74, 95\%CI:-1.01 to $-0.48, \mathrm{p}<0.01)$ were lower in norepinephrine group compared to dopamine group. The systemic vascular resistance index $(\mathrm{SMD}=1.33,95 \% \mathrm{CI}$ : 0.62 to $2.04, \mathrm{p}<0.01$ ) in norepinephrine group was higher than those of dopamine group with statistical difference. However, the MAP (SMD $=0.39,95 \%$ CI:- 0.85 to $1.63, \mathrm{p}>0.05$ ) was not statistical different between the two treatment methods, Figure 4.

\section{Publication bias}

The publications bias of the included studies were evaluated by Begg's funnel plot and Egger's line regression test. The Begg's funnel plot was left and right symmetrical, which indicated no significant publication bias (Figure 5). The Egger's line regression test also demonstrated no significant publication bias $(\mathrm{t}=-0.84, \mathrm{p}=0.425)$.

\section{Discussion}

In the present work, the Pubmed, Embase, the Cochrane Library, Web of Science, Google scholar and CNKI electronic databases were systematically searched and finally included 13 prospective clinical trials in the metaanalysis. The general methodical quality of the included 13 publications were moderate except De Backer [12] according to the Jadad scoring system. The methodical quality evaluation results indicated that the quality of the relevant clinical studies need further improvement. The pooled results showed the death risk was significant decreased ( $R R=0.89,95 \% C I: 0.81$ to $0.98, p=0.024$ ) in septic shock patients who received norepinephrine compared to those receiving dopamine, which was in accordance with the previous study [4]. The pooled results also indicated the HR, systemic vascular resistance index and cardiac index were also statistical different between norepinephrine and dopamine groups in treatment of septic shock patients $(p<0.01)$. According to our findings, norepinephrine was superior in the aspects of death risk reducing and hemodynamics compared to dopamine. The results were similar with a previous work performed by Avni et al. in year 2015[19]. Avni hand his colleges found that better hemodynamic profile and reduced adverse events rate for norepinephrine over dopamine. Norepinephrine should 


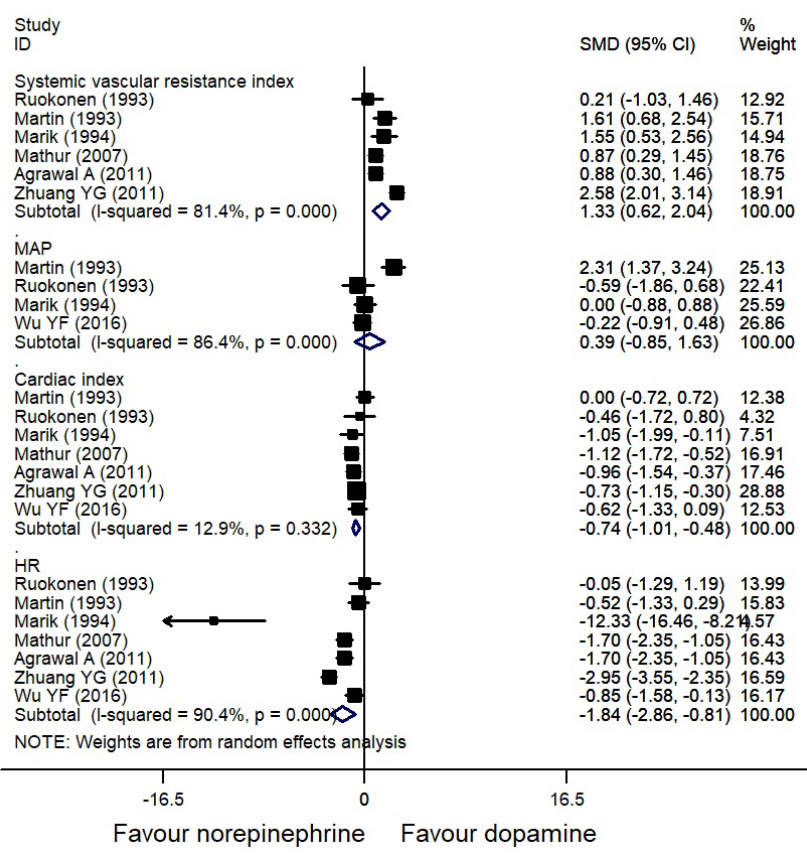

Figure 4: The forest plot of hemodynamics index for norepinephrine versus dopamine in treatment of septic shock.

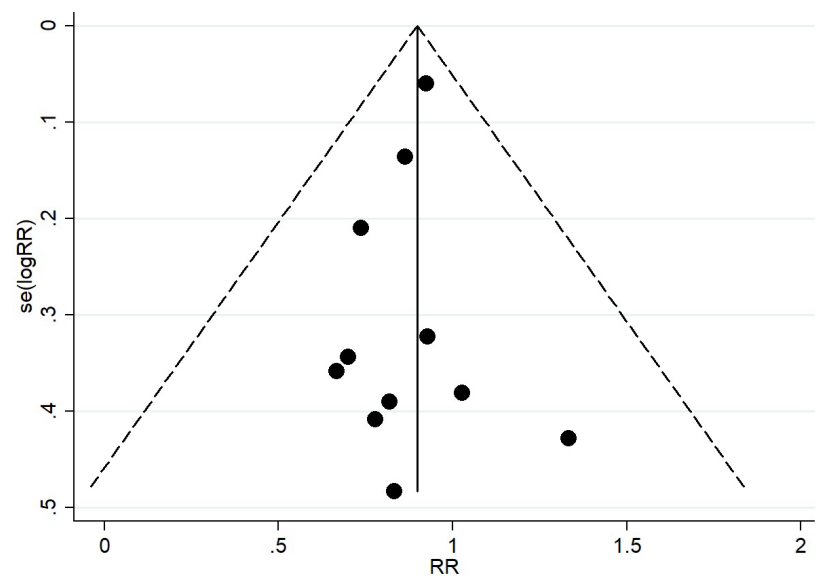

Figure 5: Begg's funnel plot in evaluation the publication bias for the include 13 studies

be regarded as the first line vasopressor in the treatment of septic shock.

Septic shock is a kind of shock caused by infection, which is characterized by abnormal blood flow distribution [20]. For patients with sepsis, once the inflammatory reaction is activated, systemic vascular resistance index is often reduced rapidly due to the expansion of arteries and veins, accompanied by the leakage of plasma into the tissue space. Thereafter the effective circulation blood volume was significant reduced, which leads to low perfusion of tissues and organs. If the above situation is not improved for a long period, the MODS would develop. Therefore, improving hemodynamics stability is one of the key factors for the success of septic shock management.

Although norepinephrine and dopamine have similar pharmacokinetics and pharmacodynamics, norepinephrine is more effective than dopamine in increasing systemic vascular resistance index and blood pressure by enhancing receptor action. Compared with dopamine, norepinephrine decreased HR and increased systemic vascular resistance index in septic shock patients, but MAP was not significant difference between the two groups.

However, in recent years, it has been reported that norepinephrine may increase the risk of immune paralysis in septic shock patients. This may lead to second infection for the septic shock subjects.

Stolk et al. [21] evaluate the potential adverse of norepinephrine for septic shock in the aspect of immune paralysis. They found that the application of norepinephrine may worsen the occurrence, degree and duration of sepsis related immune paralysis. At present, it has been confirmed that norepinephrine can produce immunosuppression and promote bacterial growth, which may increase the susceptibility to infection in vivo or animal experiments [22, 23]. However, the evidence of human beings is absent, and the effect of norepinephrine on immunosuppressant has not been confirmed clinically. Therefore, the effect of norepinephrine on the immune system in human remains to be further explored.

\section{Conclusion}

Our study included 13 prospective clinical trials and found that norepinephrine was superior in the aspects of death risk reducing and hemodynamics compared to dopamine. However, two main limitations (1)moderate methodical quality of the included studies; (2) Studies only published in English or Chinese were included) are present in our meta-analysis, which may decrease its clinical evidence. Another obvious drawback of our present work was lack of novelty compared to the previously meta-analysis relevant to this topic. Only several new publications were added in our work compared to the previous meta-analysis and the conclusion was not changed. Therefore, high quality multicenter prospective clinical trials relevant to norepinephrine versus dopamine in treatment of septic shock are needed to further validate the conclusion.

Conflict of interest: Authors state no conflict of interest 
Data Availability Statement: The datasets analyzed during the current study are available in the PubMed repository, https://pubmed.ncbi.nlm.nih.gov/; in the Embase repository, https://www.embase.com/; in the Cochrane Library repository, https://www. cochranelibrary.com/; in the Web of Science repository, www.webofknowledge.com; in the Google scholar repository, https://scholar.google.com; and in the CNKI repository, https://oversea.cnki.net/index/. The datasets generated during the current study are available from the corresponding author on reasonable request.

\section{Reference}

1. Thompson K, Venkatesh B, Finfer S. Sepsis and septic shock: current approaches to management. Intern Med J. 2019 Feb;49(2):160-70.

2. Procianoy RS, Silveira RC. The challenges of neonatal sepsis management. J Pediatr (Rio J). 2020 Mar - Apr;96 Suppl 1:80-6.

3. Varon J, Baron RM. A current appraisal of evidence for the approach to sepsis and septic shock. Ther Adv Infect Dis. 2019 Jul; https://journals.sagepub.com/ doi/10.1177/2049936119856517.

4. De Backer D, Aldecoa C, Njimi H, Vincent JL. Dopamine versus norepinephrine in the treatment of septic shock: a metaanalysis*. Crit Care Med. 2012 Mar;40(3):725-30.

5. Belletti A, Benedetto U, Biondi-Zoccai G, Leggieri C, Silvani P, Angelini GD, et al. The effect of vasoactive drugs on mortality in patients with severe sepsis and septic shock. A network metaanalysis of randomized trials. J Crit Care. 2017 Feb;37:91-8.

6. Jadad AR, Moore RA, Carroll D, Jenkinson C, Reynolds DJ, Gavaghan DJ, et al. Assessing the quality of reports of randomized clinical trials: is blinding necessary? Control Clin Trials. 1996 Feb;17(1):1-12.

7. Wu Y, Zhang N, Wu Y, Zheng Y, You X, Cao Z, et al. Effects of dopamine, norepinephrine and dobutamine on gastric mucosal pH of septic shock patients. Exp Ther Med. 2016 Aug;12(2):975-8.

8. He HW, Chen Z, Zeng WH, Yang CL. Comparison of the effect and complication between Dopamine and Norepinephrine on treatment of the septic shock. Jiangxi Med J. 2012;47(7):565-7.

9. Zhuang YG. Effects of norepinephrine and dopamine on hemodynamics and tissue oxygen metabolism in patients with septic shock. Shandong Yiyao. 2011;51(37):93-4.

10. Agrawal A, Gupta A, Consul S, Shastri P. Comparative study of dopamine and norepinephrine in the management of septic shock. Saudi J Anaesth. 2011 Apr;5(2):162-6.

11. Wu JF, Chen W, Ou YB, Yang CH, Chen MY, Huang, SW, et al. Effect of dopamine and norepinephrine on hemodynamics and oxygen metabolism of tissue in patients with septic shock [Electronic Version]. Chin Arch Gen Surg. 2010;4(2):117-21.

12. De Backer D, Biston P, Devriendt J, Madl C, Chochrad D, Aldecoa C, et al.; SOAP II Investigators. Comparison of dopamine and norepinephrine in the treatment of shock. N Engl J Med. 2010 Mar;362(9):779-89.
13. Liu P, Chen TE, Zhang YC, Yang Q, Chen PF, Hu XJ, et al. Comparison evaluation of resuscitation effect of norepinephrine and dopamine on the treatment of septic shock. Clin Educ Gen Pract. 2010,(3):265-7.

14. Patel GP, Grahe JS, Sperry M, Singla S, Elpern E, Lateef O, et al. Efficacy and safety of dopamine versus norepinephrine in the management of septic shock. Shock. 2010 Apr;33(4):375-80.

15. Gao JL, Li XL, Wang AT. Effects of dopamine and norepinephrine on renal perfusionin patients with septic shock. Hebei Med J. 2008;(8):1118-20.

16. Marik PE, Mohedin M. The contrasting effects of dopamine and norepinephrine on systemic and splanchnic oxygen utilization in hyperdynamic sepsis. JAMA. 1994 Nov;272(17):1354-7.

17. Martin C, Papazian L, Perrin G, Saux P, Gouin F. Norepinephrine or dopamine for the treatment of hyperdynamic septic shock? Chest. 1993 Jun;103(6):1826-31.

18. Ruokonen E, Takala J, Kari A, Saxén H, Mertsola J, Hansen EJ. Regional blood flow and oxygen transport in septic shock. Crit Care Med. 1993 Sep;21(9):1296-303.

19. Avni T, Lador A, Lev S, Leibovici L, Paul M, Grossman A. Vasopressors for the Treatment of Septic Shock: Systematic Review and Meta-Analysis. PLoS One. 2015 Aug;10(8):e0129305.

20. Dellinger RP, Levy MM, Rhodes A, Annane D, Gerlach H, Opal SM, et al.; Surviving Sepsis Campaign Guidelines Committee including The Pediatric Subgroup. Surviving Sepsis Campaign: international guidelines for management of severe sepsis and septic shock, 2012. Intensive Care Med. 2013 Feb;39(2):165228.

21. Stolk RF, van der Poll T, Angus DC, van der Hoeven JG, Pickkers $P$, Kox M. Potentially Inadvertent Immunomodulation: Norepinephrine Use in Sepsis. Am J Respir Crit Care Med. 2016 Sep;194(5):550-8.

22. Martino M, Rocchi G, Escelsior A, Fornaro M Immunomodulation Mechanism of Antidepressants: Interactions between Serotonin/Norepinephrine Balance and Th1/Th2 Balance. Curr Neuropharmacol. 2012 Jun;10(2):97-123.

23. Chen H, Wang L, Zhou Z, Hou Z, Liu Z, Wang W, et al. The comprehensive immunomodulation of NeurimmiRs in haemocytes of oyster Crassostrea gigas after acetylcholine and norepinephrine stimulation. BMC Genomics. 2015 Nov;16(1):942. 